\title{
CHIRONOMUS Newsletter now compliant with the International Code of Zoological Nomenclature
}

\author{
Torbjørn Ekrem \\ Co-editor Chironomus Newsletter on Chironomidae Reseach
}

Museum of Natural History and Archaeology, Norwegian University of Science and Technology, Trondheim, Norway.E-mail: torbjorn.ekrem@ntnu.no

It is a pleasure to inform you that as of this issue, the newsletter complies with article 8.6 of the International Code of Zoological Nomenclature. This means that texts published in the newsletter are to be regarded as a published work also for the purposes of zoological nomenclature, and that we now welcome papers that involve nomenclatorial acts. In order to comply with article 8.6, copies of the newsletter must be deposited in at least five major publicly accessible libraries. This and future issues of the CHIRONOMUS Newsletter will therefore be printed in a limited number of paper copies and distributed to the libraries listed on page two of this issue. We are very pleased that all libraries we contacted were willing to receive and store copies of the CHIRONOMUS Newsletter and look forward to receive more taxonomy manuscripts for our Current Research section. 